\title{
Hough Transform Based Pattern Recognition for the PANDA Forward Tracking System
}

\author{
Martin J. Galuska*(1) \\ Yutie Liang $^{(1)}$, David Münchow ${ }^{(1)}$, Stefano Spataro(2) ${ }^{(2 j o ̈ r n ~ S p r u c k}{ }^{(1)}$ and \\ Milan Wagner $^{(1)}$ for the PANDA Collaboration
}

(1) Justus-Liebig-Universität Gießen II. Physikalisches Institut

Heinrich-Buff-Ring 16

D-35392 Gießen
(2) Universita' di Torino Dipartimento di Fisica Via P. Giuria, 1

I-10125 Torino

The planned PANDA fixed-target experiment will produce up to $2 \cdot 10^{7}$ antiproton-proton or antiproton-nucleus collisions per second. Up to 8 primary charged particles per event are expected to reach the acceptance of the PANDA Forward Tracking System (FTS) detector. Due to a drift time of $\leq 150 \mathrm{~ns}$ for the FTS straw tubes the signals of on average 3 events will overlap in this layered detector at the peak interaction rate of $20 \mathrm{MHz}$. The number of overlapping events is $\leq 8$ with a probability of $\geq 99.6 \%$.

In this paper we describe a Hough transform based charged particle tracking algorithm for the $\bar{P} A N D A$ FTS detector. In the region of the PANDA Forward Spectrometer $(290 \mathrm{~cm} \leq z \leq$ $780 \mathrm{~cm}$ with the $z$-axis pointing into the beam direction) we use a 3-stages track model of line+parabola+line for the projection of the track into the bending $x$-z-plane. The projection into the non-bending $y$-z-plane is approximated by a line for this region.

Preliminary results for single particle momentum resolutions obtained with a proof-of-concept implementation of the described algorithm are presented. Simulations were carried out in PandaRoot, the official framework for simulation, reconstruction and analysis for the PANDA experiment.

This work was supported in part by BMBF (05P12RGFPF), HGS-HIRe for FAIR and the LOEWE-Zentrum HICforFAIR.

51st International Winter Meeting on Nuclear Physics

21-25 January 2013

Bormio (Italy)

* Speaker.

${ }^{\dagger}$ E-mail: Martin.J.Galuska@physik.uni-giessen.de 


\section{The PANDA Experiment}

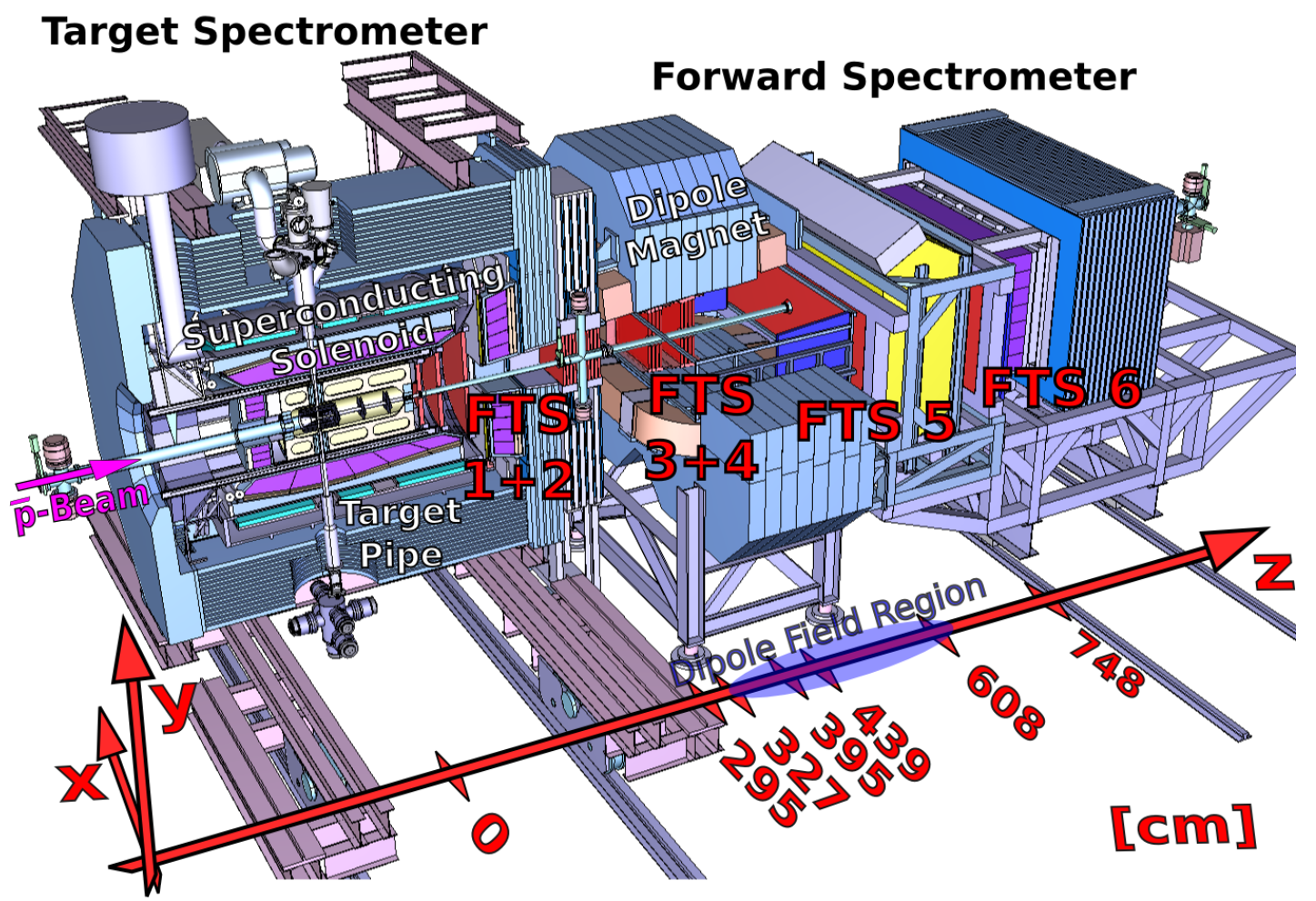

Figure 1: The FTS is part of the PANDA Forward Spectrometer and measures the deflection of charged particles in the magnetic field of the dipole magnet. Its six stations are depicted by red boxes in the picture. The corresponding distances to the interaction point in $z$-direction are indicated.

The PANDA (anti-Proton ANnihilations at DArmstadt) experiment is designed to investigate $\overline{\mathrm{p}}+\mathrm{p}$ and $\overline{\mathrm{p}}+A$ collisions with various internal proton $(\mathrm{p})$ or nuclear $(A)$ targets and an anti-proton $(\overline{\mathrm{p}})$ beam in the momentum range from 1.5 to $15 \mathrm{GeV} / \mathrm{c}$ with excellent beam momentum definition [1]. It is a part of the FAIR [2,3] (Facility for Anti-proton and Ion Research) extension of the existing GSI Helmholtz Centre for Heavy Ion Research facility in Darmstadt, Germany and is scheduled to start operation in 2018.

The PANDA Physics program focuses on accurate measurements in the non-perturbative regime exploring the nature of the strong interaction and progressing the understanding of the QCD spectrum and hadron structure. [1] It will provide information to questions regarding confinement, in-medium modifications of hadron masses, the structure of the nucleon, spin degrees of freedom and the search for color neutral objects. Its measurement capabilities range from meson spectroscopy (D mesons, charmonium, glueballs, hybrids, tetraquarks, molecules), charmed and multi-strange baryon spectroscopy and electromagnetic processes $\left(\mathrm{p} \overline{\mathrm{p}} \rightarrow \mathrm{e}^{+} \mathrm{e}^{-}, \mathrm{p} \overline{\mathrm{p}} \rightarrow \gamma \gamma\right.$, DrellYan) to measurements of properties of single and double hypernuclei and of hadrons in nuclear matter [1, 4]. The combination of a phase-space cooled antiproton beam with beam momentum resolutions of $\Delta p_{\text {beam }} / p_{\text {beam }} \leq 4 \cdot 10^{-5}$ and dense internal targets will offer unique possibilities 
for studies of numerous aspects of the strong interaction. Unlike in electron-positron machines $\bar{P} A N D A$ can produce charmonium(-like) states with arbitrary non-exotic quantum numbers exclusively which allows resonance scans of such states with unprecedented precision [5].

The technical requirements for the PANDA detector are quite high: its readout needs to be capable of handling rates up to $2 \cdot 10^{7}$ interactions per second with a versatile event selection. In order to achieve nearly $4 \pi$ acceptance and good resolution for charged particles with a large momentum range the detector is comprised of a target and a forward spectrometer as shown in figure 1. Both units feature charged particle identification, tracking and electromagnetic calorimetry "to allow to detect the complete spectrum of final states relevant for the PANDA physics objectives" [1].

The target spectrometer surrounds the interaction point and detects particles at high polar angles $5^{\circ} \leq \theta \leq 170^{\circ}$ to the beam-axis $z$. The momentum resolution in the target spectrometer relies on a $2 \mathrm{~T}$ magnetic field provided by a superconducting solenoid. As PANDA is a fixed target experiment a large fraction of decay particles will be boosted toward forward angles. To account for this a dedicated forward spectrometer supplements the target spectrometer. The forward spectrometer is located downstream of the interaction region and measures charged particle tracks at small polar angles $\theta$ below $5^{\circ}$ in the vertical and $10^{\circ}$ in the horizontal plane. Its magnetic field with a maximum bending power of $2 \mathrm{Tm}$ is provided by a dipole magnet. As the deflection of the antiproton beam in the beam line caused by the dipole field can only be corrected within certain limits, the magnetic field strength will have to be adjusted according to the beam momentum. For further details see [6].

\section{The PANDA Forward Tracking System (FTS)}

The PANDA Forward Tracking System is a key part of the Forward Spectrometer. The detector measures the deflection of charged particles in the magnetic field of the dipole magnet. It is comprised of a total of six stations with four straw tube double layers each, two of which have vertical wires, the other two have wires inclined by $\pm 5^{\circ}$. Chambers 1 and 2 will be placed in front, 3 and 4 within, 5 and 6 behind the dipole magnet (see fig. 1). This configuration will allow to track charged particles with a large range of momenta down to very low momentum particles whose trajectories might curl up inside the magnetic field. The momentum acceptance extends above 3\% of the beam momentum.

The maximum drift time in the straw tubes of the FTS is approximately $130 \mathrm{~ns}$ for a gas mixture of $\mathrm{Ar}+\mathrm{CO}_{2}(90: 10)$ at a pressure of 2 bar and an anode wire voltage of $1800 \mathrm{~V}$ [7]. This number should hold for the four tracking stations which are located in a relatively low magnetic field. For the stations 3 and 4 inside the dipole gap, however, the maximum drift time can increase up to about $150 \mathrm{~ns}$ if the dipole magnet is adjusted to the highest beam momentum $p_{\text {beam }}=15 \mathrm{GeV} / \mathrm{c}$ [8]. The increase in drift time is caused by the Lorentz force: the trajectories of electrons drifting towards anode wires are not straight in the presence of a magnetic field oriented along the straw tubes. The electrons rather drift along spirals to the wires.

\section{Reconstruction of Charged Particles from Detector Hit Information}

The reconstruction of charged particle tracks from tracking detector signals can be divided 
into two steps, usually called track finding (or pattern recognition) and track fitting. In the pattern recognition stage subsets of measured detector hits are combined to track candidates. The track fitting stage determines whether a given track candidate is acceptable and refines its parameters [9].

The most straight forward method of pattern recognition is a combinatorial method which forms track candidates from all combinations of hits that the detector registered in a given time window. For a detector consisting of $l$ layers and for approximately $N$ hits per layer coming from $N$ tracks present in a given time window, the combinatorial method reconstructs on the order of $N^{l}$ track candidates. Six FTS stations with four double layers each result in 24 double layers in the PANDA FTS case. For a numerical example we assume 8 charged tracks per event within the acceptance of the FTS and 6 time-wise overlapping events ${ }^{1}$ within a 150 ns time-window resulting in 48 charged tracks which need to be taken into account. The combinatorial method applied in this case would result with on the order of $48^{24} \approx 2.2 \cdot 10^{40}$ track candidates. Analysing such a large number of track candidates far exceeds the computing power available for the PANDA FTS online tracking.

In order to significantly lower the number of track candidates a priori knowledge of the track geometry can be used. Two methods are usually distinguished: Local and global methods [10].

Local methods such as track following process all tracks independently of each other. Initial track candidates are formed from a small number of hits, additional ones can be added using interor extrapolation. If the utilized algorithms find more than a certain threshold of hits which could belong to a given track candidate, the candidate is passed to the fitting stage, otherwise it is discarded. Global methods treat all hits in the same way. The algorithm studied in this paper uses a global method to find tracklets which are short track pieces and uses ideas typical for local methods to combine the tracklets to track candidates.

\section{Hough Transform Based Pattern Recognition Algorithm}

Global track finding methods can be regarded as transformations of coordinate space hits into a mathematical space in which the track candidates can be found more conveniently. The Hough transform [11] is an example for a global method. It uses a parameter space whose dimensions are the parameters of the underlying track model. Each point in the parameter space describes a track in coordinate space. This track is obtained by plugging in the corresponding parameters into the equation for the utilized track model. When performing a Hough transform each detector hit "votes" for all tracks which intersect with the hit location in coordinate space. The parameter space is discretized and each bin counts the number of votes it receives from the detector hits. The result of a Hough transform therefore is a discretized density distribution in parameter space which is also refered to as a "Hough space". Finding track candidates based on hit information corresponds to finding local maxima in the Hough space. The coordinates of local maxima describe the most likely track candidates based on the track model and the measured detector hits. The main advantages of Hough transforms are their robustness against missing, additional and noisy hits as well as their suitability for an implementation in GPU or FPGA hardware for online tracking [12].

Figure 2 shows the basic idea of a Hough transform for a simple toy detector consisting of four layers of active material. Charged particles originate from the interaction point, travel through

\footnotetext{
${ }^{1}$ The probability for $\leq 6$ overlapping events is $96.6 \%$ for an average time of 50 ns between two events.
} 
a magnetic field and leave hits in the active material of the detector. The top row of pictures shows views of the detector and the tracks in coordinate space, on the bottom we illustrate the parameter space for a parabola track model. The track model requires two parameters: $\theta$ is the angle of the track toward the $z$-axis in the interaction point, $1 / p_{z x}$ is the inverse of the particle's momentum in the $x$-z-plane.

The picture on the left illustrates the fact that one track in coordinate space corresponds to one point in parameter space. We want to find the depicted track based on the registered hits in the toy detector. The picture in the middle shows a hit which the sought-after track left in the third detector layer. When the Hough transform processes that hit, it "votes" for all tracks that could cause such a hit and satisfy the parabola track model with apex in the origin. In the parameter space all points are marked which receive a vote from the hit. For simplicity, we assume all these points lie on a straight line and we neglect the discretization of the parameter space. The picture on the right shows the result of the Hough transform after processing four hits. Each hit "voted" for a parameter space curve. Each point on these lines is a paramter pair $\left(\theta, 1 / p_{z x}\right)$ which corresponds to a track that could leave at least one of the hits. The intersection of the four curves in parameter space represents the track which can leave all four hits in the detector and therefore is the most likely track candidate given the detector hit information and the track model.



Figure 2: Illustration of the basic principle for a Hough transform using a parabola track model for a toy detector. This figure is based on figure 2 in [12].

In principle, any analytical expression can be used as a track model in a Hough transform. We choose a multi-stage track model for the tracks inside the PANDA FTS region which fits the detector's geometry: for the region before $(290 \mathrm{~cm} \leq z \leq 368 \mathrm{~cm})$ and after the dipole field $(605 \mathrm{~cm} \leq z \leq 780 \mathrm{~cm})$ we approximate the tracks' projections in the $x$-z-plane with straight lines. Charged particle tracks are bent in the region of the dipole field $(368 \mathrm{~cm} \leq z \leq 605 \mathrm{~cm})$ in the $x$-z-plane. We therefore assume a parabolic track model in that region according to equation (4.2) which describes a rotated parabola similar to the track model studied in [12].

In the forward region before and after the dipole field the tracks can be described by straight lines in the $x$-z-plane according to equation 4.1

$$
x_{\text {hit }}=\tan \theta \cdot\left(z_{\text {hit }}-z_{0}\right)+x_{0}
$$


in which $\theta$ is the angle between the line and the $z$-axis in this plane, $x_{0}$ is the intercept at $z$ coordinate $z_{0}$ and $\left(x_{\text {hit }}, z_{\text {hit }}\right)$ are the coordiantes of hits in non-skewed FTS straws.

The amount by which the tracks are bent by the dipole field depends on the projection $p_{z x}$ of the track's momentum $p$ into the $x$-z-plane, the peak strength of the $y$-component of the magnetic field $B_{y}$, the particle's charge $Q$ and the angle $\theta$ of the track toward the $z$-axis in the $x$ - $z$-plane at the point of entry to the Forward Spectrometer. We search for a parabola for every line we find on hits in stations 1 and 2. Each of these lines determines the possible range for the parabola's rotation $\theta$ and fixes the coordinates of its apex $\left(x_{0}, z_{0}\right)$. If the existence of such a parabola is consistent with the hits on the stations 3,4 and 5, its stretch factor $1 / p_{z x}$ is adjusted according to equation (4.2). $(x, z)=\left(x_{\text {hit }}-x_{0}, z_{\text {hit }}-z_{0}\right)$ denominate the coordiantes of hits in non-skewed FTS straws relative to the apex.

$$
\frac{1}{p_{z x}}=\frac{2 \cdot(x \cdot \cos \theta-z \cdot \sin \theta)}{Q \cdot B_{y} \cdot(z \cdot \cos \theta+x \cdot \sin \theta)^{2}}
$$

Finally, in the $y$-z-plane we use a straight line for the entire region of the FTS $(290 \mathrm{~cm} \leq z \leq$ $780 \mathrm{~cm}$ ). The necessary two-dimensional Hough transforms are carried out according to the following algorithm:

Straight lines in the $x$-z-plane before and after the dipole field: For each fired non-skewed straw in the stations $1+2$ and $5+6$ the drift time information is used to determine the set of possible hit $x$ - and $z$-coordinates. Two separate Hough transforms are performed on these hit candidates to find two sets of straight lines in the region of the Forward Spectrometer; one set before and one after the dipole field. The necessary Hough transforms can be performed in parallel.

The straight lines in the $x$-z-plane before and after the dipole field are used as initial track candidates. Additionally, the $x$ - and $z$-coordinates of hits in non-skewed straws in stations $1+2+5+6$ are determined in this first step of the algorithm.

Adding skewed hits to lines: The lines which were found in the previous step are expanded to three-dimensional planes. The points of closest approach are calculated for all combinations of fired skewed straws from two neighboring double layers within the same station. If their connecting line intersects with one of the planes, the point of intersection is added to the corresponding track candidate as a hit candidate. If it is not possible to find a reasonable amount of such hit candidates, the corresponding track candidate is rejected.

This step is needed to make $(x, z)$-coordiante information available for all hits in the chambers $1+2$ and $5+6$. Additionally, the $y$-positions for hits from skewed straws become available.

Parabola in the $x$-z-plane within the dipole field: For each remaining track candidate which was built up from station 1 and 2 hits a parabola Hough transform in the $x$-z-plane is performed. The line from step 1 determines the shift of the parabola's apex in $x$-direction and its $\theta$-angle restricts the corresponding angle for the parabola. This ensures a smooth link between the line and the parabola. Only hits from non-skewed straws in chambers $3-5$ are used for the parabola Hough transform. Drift circles are taken into account. If this step cannot find a parabola which matches the corresponding line, the track candidate is rejected. 
The track candidates which pass this filtering step and have sufficient momenta are matched with a line from station 5 and 6 hits.

The parabola Hough transform delivers information on a given track candidate's charge and an estimate of $p_{x}$ and $p_{z}$, the momentum components of the charged particle at the entrance to the FTS.

Adding skewed hits to parabolas: The parabolas which were found in the previous step are expanded into three dimensional point sets. For all combinations of fired skewed straws from neighboring double layers in stations 3 to 5 the points of closest approach are calculated. For two corresponding points of closest approach their connecting line is checked for intersection with one of the expanded parabolas. If it exists, the point of intersection is added to the corresponding track candidate as a hit candidate. If not enough hit candidates are found, the track candidate corresponding to the parabola is discarded.

This step makes all spacial information for the hits from skewed straws in stations 3 and 4 available.

Straight lines in the $y$-z-plane: A straight line Hough transform in the non-bending $y$-z-plane is performed on all hits from station $1-6$ belonging to a given track candidate. The hits with unknown $y$-coordinate are required to lie on the found line in the $y$-z-plane.

Performing this step makes the $y$-information of all hits in all chambers available. Additionally, $p_{y}$ at the entrance to the FTS can be reconstructed.

After the algorithm completed the above steps, the hit coordinates of all hits in the FTS are reconstructed. The charge and all momentum components are estimated for the track candidates at the position of the entrance to the FTS.

\section{Preliminary Results}

The preliminary momentum resolution was studied with a proof-of-concept implementation of the pattern recognition algorithm described in section 4. This implementation did not yet use any drift time information, none of the hits in station 6 and neither any hit information from skewed straws in chambers 1 to 5 . As the skewed hits provide the only information for a reconstruction of $y$-coordinates, the implementation only considered the projection of tracks into the $x$-z-plane. It performed a line Hough transform on the hits from non-skewed straws in stations 1 and 2 and used the obtained parameters for a corresponding parabola Hough transform on the non-skewed hits from stations 3 to 5 . The momentum information was extracted from the parameters found by the parabola Hough transform. A matching with other detectors was not done, additional fitting was not applied. The results merely represent the resolution obtained with the partial information used in the proof-of-concept implementation of the patter recognition algorithm.

In figure 3 the relative momentum resolution $\frac{p_{z, \mathrm{rec}}-p_{z, \mathrm{MC}}}{p_{z, \mathrm{MC}}}$ is plotted against the $z$-component $p_{z, \mathrm{MC}}$ of the Monte Carlo truth momentum $p_{\mathrm{MC}} \cdot p_{z, \text { rec }}$ designates the $z$-component of the reconstructed track momentum. $5 \cdot 10^{4}$ single muon events with $0.5 \leq p_{\mathrm{MC}} \leq 5.0 \mathrm{GeV} / \mathrm{c}$ were simulated with PandaRoot. In momentum slices of $50 \mathrm{MeV} / \mathrm{c}$ the relative $p_{z}$ resolution was fitted by a Gaussian distribution. The corresponding standard deviations are shown in the plot. All tracks which 


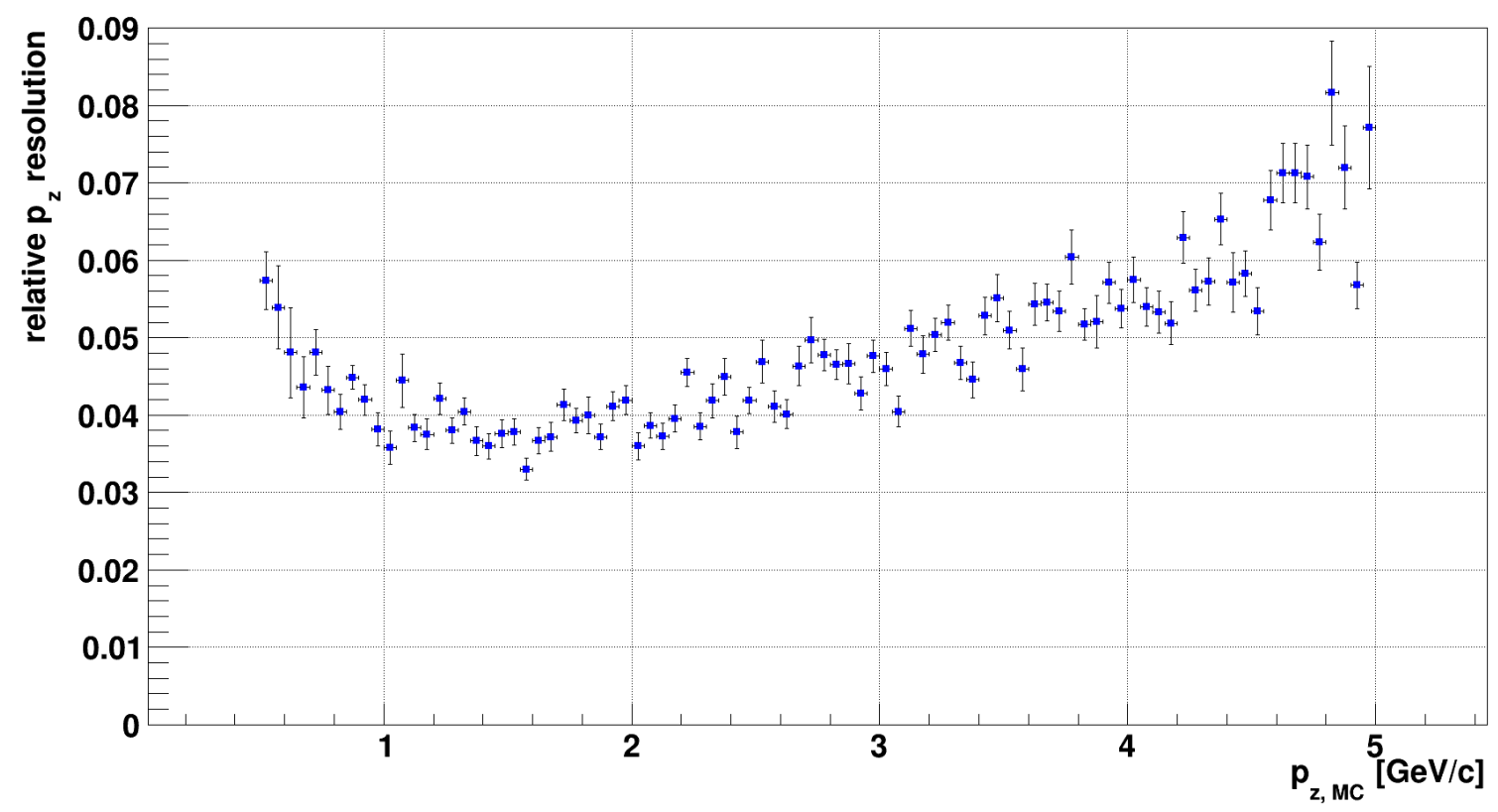

Figure 3: The standard deviations of Gaussian fits to the relative momentum distributions $\frac{p_{z, \mathrm{rec}}-p_{z, \mathrm{MC}}}{p_{z, \mathrm{MC}}}$ for momentum slices of $50 \mathrm{MeV} / \mathrm{c}$ width obtained by the PANDA FTS pattern recognition are plotted against the $z$-component $p_{z, \mathrm{MC}}$ of the Monte Carlo truth momentum $p_{\mathrm{MC}}$.

have at least two (out of a maximum of eight) hits in non-skewed straws in FTS stations $1+2$ and four (out of a maximum of twelve) hits in FTS stations 3 to 5 are considered to be within the detector's acceptance in this study.

All $\bar{P} A N D A$ detectors were included in the simulation. The dipole field was set according to $p_{\text {beam }}=15 \mathrm{GeV} / \mathrm{c}$. Multiple scattering and energy losses were simulated. The angle towards the $z$-axis (in the interaction point) was uniformly distributed in the region $0.1^{\circ}<\theta<5^{\circ}$. All polar angles $0^{\circ} \leq \phi<360^{\circ}$ were allowed. The obtained results show that the algorithm idea has sufficient potential to be further investigated.

Figure 4 shows an example single muon event with $p_{z}=1.233 \mathrm{GeV} / \mathrm{c}$ which was processed with the proof-of-concept implementation. The reconstructed momentum extracted from the parabola Hough transform matches the input momentum closely. As hits (represented by black crosses) the wire positions of fired non-skewed straws are used. The red stars mark Monte Carlo points which are plotted to give a qualitative impression of the match between the true and the reconstructed particle track plotted in blue. The algorithm does not use the Monte Carlo truth information.

The profile of the magnetic field strength is plotted in the background of figure 4. The region of low magnetic field strengths is the "line region", the "parabola region" corresponds to a stronger field. The tracklet in the "line region" was found from station $1+2$ hits in the discretized parameter space density distribution shown in the left plot of figure 5. This tracklet represents the most likely line based on the track model and the detector hits. It determines the apex of a matching parabola which is then found from stations 3 to 5 hits in the Hough space shown in the right plot of figure 5. 


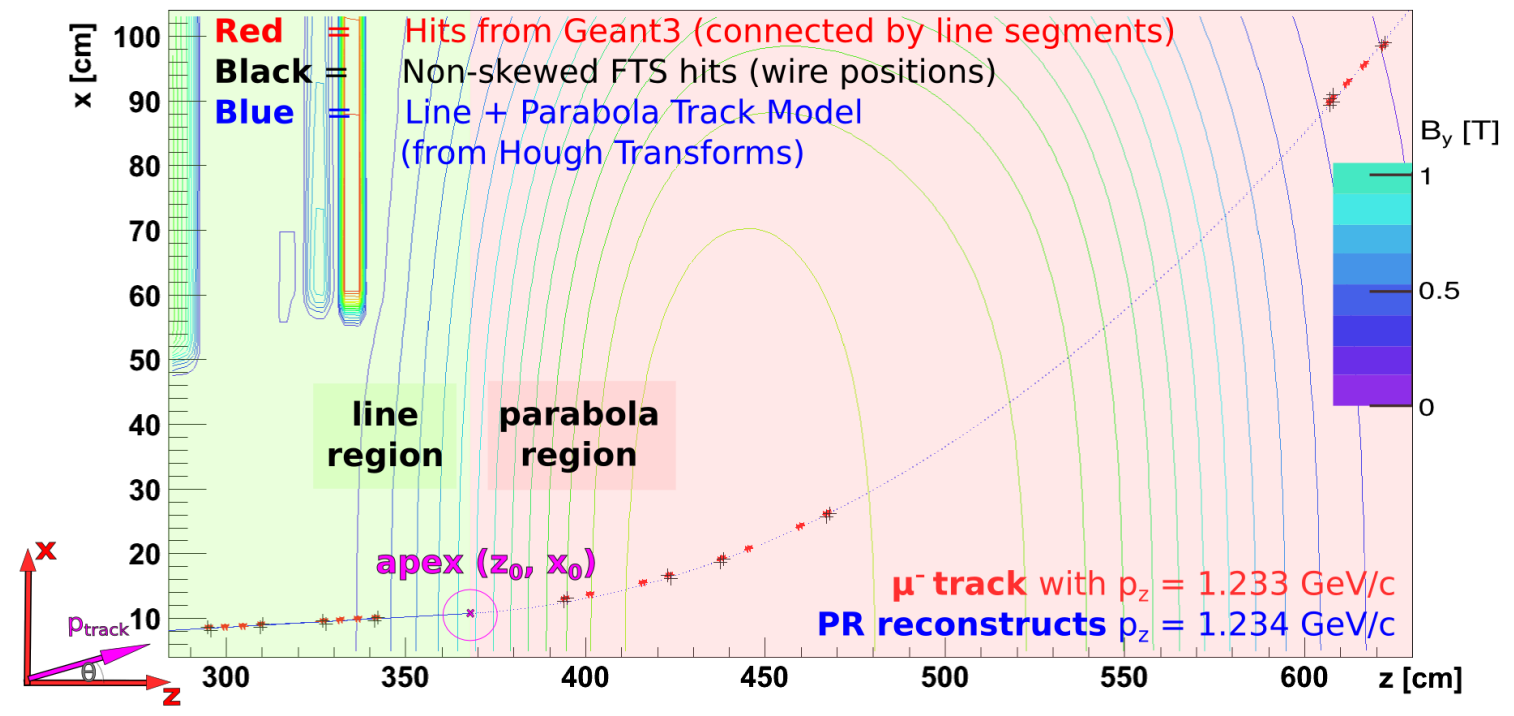

Figure 4: Example event processed with a proof-of-concept implementation of the algorithm described in section 4.
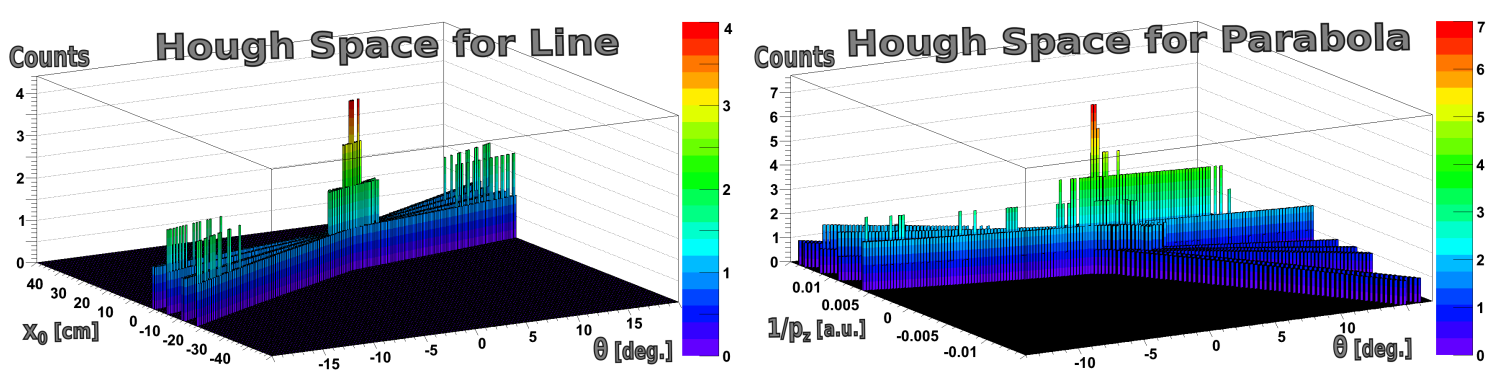

Figure 5: The parameter spaces for a line and for a parabola Hough transform are shown. Both histograms were produced by the proof-of-concept implementation during the processing of the example event shown in figure 4 in order to determine the most likely track candidate.

\section{Summary and Outlook}

The goal of the work presented in this paper is the design of a pattern recognition algorithm for the PANDA FTS detector. As the algorithm should be applicable to both offline and online tracking, a global method based on Hough transforms was chosen to be studied. The current status is presented in form of a complete algorithm alongside a description of a proof-of-concept implementation. First momentum resolution results using only a fraction of the available information are promising.

The milestones for further developments foresee the finalization and test of a full algorithm implementation. The algorithm's time and memory consumption will be optimized using an adaptive Hough transform [13] approach. A method for finding the event start time using only information available online to the FTS pattern recognition will be tested. The drift times of signals will be included and the algorithm for the reconstruction of $y$-information will be added. Studies of effi- 
ciency, ghost rate and momentum resolution for multi-particle events will determine how well the algorithm performs under realistic conditions after a timebased simulation [14] for the FTS is implemented. It is foreseen to study alternative algorithms for FTS pattern recognition and choose the best-performing to refine the detector's geometry. The matching of track candidates found in the FTS with candidates from other relevant detectors will need careful attention due to the magnetic fields from the superconducting solenoid in the Target Spectrometer region with $B_{z}=2 \mathrm{~T}$ and the beam momentum dependent downstream dipole magnet of up to $2 \mathrm{Tm}$ bending power.

\section{References}

[1] The $\bar{P} A N D A$ Collaboration, "Physics Performance Report for PANDA: Strong Interaction Studies with Antiprotons," arXiv:0903.3905 [hep-ex], 2009.

[2] GSI Helmholtzzentrum für Schwerionenforschung, "FAIR - An International Accelerator Facility for Beams of Ions and Antiprotons, Baseline technical report," http://www. fair-center.de/ de/fuer-nutzer/publikationen/fair-publikationen.html, 2006.

[3] GSI Helmholtzzentrum für Schwerionenforschung, "FAIR Green Paper - The Modularized Start Version," http://www.fair-center.de/fileadmin/fair/publications_FAIR/ FAIR_GreenPaper_2009.pdf, 2009.

[4] J. Ritman, "Status of PANDA," in $8^{\text {th }}$ International Workshop on Heavy Quarkonium, 2011.

[5] M. J. Galuska, W. Kühn, J. S. Lange, and B. Spruck, "Results for a Simulated Resonance Scan of the X(3872) at PANDA," Proceedings of Science, vol. PoS(Bormio2012)018, 2012.

[6] The PANDA Collaboration, "Technical Design Report for the PANDA Solenoid and Dipole Spectrometer Magnets," arXiv:0907.0169 [physics.ins-det], 2009.

[7] J. Smyrski, "Geometry of the Forward Tracking Stations." Internal document, 2009.

[8] J. Smyrski, "Private communication." 2013.

[9] H. Grote, "Pattern recognition in high-energy physics," Reports on Progress in Physics, vol. 50, no. 4, p. 473, 1987.

[10] R. Mankel, "Pattern recognition and event reconstruction in particle physics experiments," Reports on Progress in Physics, vol. 67, no. 4, p. 553, 2004.

[11] P. V. C. Hough, "Method and means for recognizing complex patterns," U.S. Patent 3069654, 1962.

[12] J. Gläss, C. Steinle, and R. Männer, "Tracking in the Silicon Tracker System of the CBM Experiment using Hough Transform," 14th IEEE-NPSS REAL TIME Conference, 2005.

[13] J. Illingworth and J. Kittler, "The Adaptive Hough Transform," IEEE Transactions on Pattern Analysis and Machine Intelligence, vol. 9, no. 5, pp. 690-698, 1987.

[14] T. Stockmanns, "Time based simulation," in Panda Computing Week, 2012. 